\title{
RIGIDITY OF COMPLETE ENTIRE SELF-SHRINKING SOLUTIONS TO KÄHLER-RICCI FLOW
}

\author{
GREGORY DRUGAN, PENG LU, AND YU YUAN
}

\begin{abstract}
We show that every complete entire self-shrinking solution on complex Euclidean space to the Kähler-Ricci flow must be generated from a quadratic potential.
\end{abstract}

\section{INTRODUCTION}

In this note, we prove the following result.

Theorem 1.1. Suppose $u$ is an entire smooth pluri-subharmonic solution on $\mathbb{C}^{m}$ to the complex Monge-Ampère equation

$$
\ln \operatorname{det}\left(u_{\alpha \bar{\beta}}\right)=\frac{1}{2} x \cdot D u-u .
$$

Assume the corresponding Kähler metric $g=\left(u_{\alpha \bar{\beta}}\right)$ is complete. Then $u$ is quadratic.

Any solution to (1.1) leads to a self-shrinking solution $v(x, t)=-t u(x / \sqrt{-t})$ to a parabolic complex Monge-Ampère equation

$$
v_{t}=\ln \operatorname{det}\left(v_{\alpha \bar{\beta}}\right)
$$

in $\mathbb{C}^{m} \times(-\infty, 0)$, where $z^{\alpha}=x^{\alpha}+\sqrt{-1} x^{m+\alpha}$. Note that the above equation of $v$ is the potential equation of the Kähler-Ricci flow $\partial_{t} g_{\alpha \bar{\beta}}=-R_{\alpha \bar{\beta}}$. In fact, the corresponding metric $\left(u_{\alpha \bar{\beta}}\right)$ is a shrinking Kähler-Ricci (non-gradient) soliton.

Assuming a certain inverse quadratic decay of the metric-a specific completeness assumption-Theorem 1.1 has been proved in $\mathrm{CCY}$. Similar rigidity results for selfshrinking solutions to Lagrangian mean curvature flows were obtained in [HW], $\mathrm{CCY}]$, and [DX].

The idea of our argument, as in [CCY], is still to force the phase $\ln \operatorname{det}\left(u_{\alpha \bar{\beta}}\right)$ in equation (1.1) to attain its global maximum at a finite point. As this phase satisfies an elliptic equation without the zeroth order terms (see (2.2) below), the strong maximum principle implies the constancy of the phase. Consequently, the homogeneity of the self-similar terms on the right hand side of equation (1.1) leads to the quadratic conclusion for the solution.

However, the difficulty of the above argument lies in the first step: Here we cannot construct a barrier as in $[\mathrm{CCY}]$, which requires the specific inverse quadratic decay of the metric, to show the phase achieves its maximum at a finite point. The new observation is that the radial derivative of the phase, which is the negative of the scalar curvature of the metric (2.3), is in fact nonpositive; hence the phase

This work was supported by the National Science Foundation [RTG 0838212 to G.D., DMS 1100966 to Y.Y.]; and the Simons Foundation [229727 to P.L.]. 
value at the origin is its global maximum. The nonnegativity of the scalar curvature is a result of B.-L. Chen [Ch], as the induced metric $g(x, t)=\left(u_{\alpha \bar{\beta}}(x / \sqrt{-t})\right)$ is a complete ancient solution to the (Kähler-)Ricci flow. Here we provide a direct elliptic argument for the nonnegativity of the scalar curvature for the complete selfshrinking solutions (in Section 3, after necessary preparation in Section 2, where a pointwise approach to Perelman's upper bound of the Laplacian of the distance $\mathrm{P}$ is also included). Heuristically one sees the minimum of the scalar curvature is nonnegative from its inequality (2.5); it is definitely so if the minimum is attained at a finite point. Note that a thorough study of the lower bound of scalar curvatures of the gradient Ricci solitons is presented in [CCG4, Chap.27].

\section{Preliminary Results}

For the potential $u$ of the Kähler metric $g=\left(g_{\alpha \bar{\beta}}\right)=\left(u_{\alpha \bar{\beta}}\right)$ on $\mathbb{C}^{m}$, we denote the phase by $\Phi=\ln \operatorname{det}\left(u_{\alpha \bar{\beta}}\right)$. Then the Ricci curvature is given by $R_{\alpha \bar{\beta}}=-\frac{\partial^{2} \Phi}{\partial z^{\alpha} \partial \bar{z}^{\beta}}$. The "complex" scalar curvature is $R=g^{\alpha \bar{\beta}} R_{\alpha \bar{\beta}}$ ( $R$ is one-half of the usual "real" scalar curvature). Let $\rho(x)$ denote the Riemannian distance from $x$ to 0 in $\left(\mathbb{C}^{m}, g\right)$. For a solution $u$ of (1.1), we derive the following equations and inequalities for those geometric quantities.

2.1. Equation for phase $\Phi$. Since $u$ is a solution of (1.1), the phase satisfies the equation $\Phi=\frac{1}{2} x \cdot D u-u$. Taking two derivatives,

$$
-R_{\alpha \bar{\beta}}=\frac{\partial^{2} \Phi}{\partial z^{\alpha} \partial \bar{z}^{\beta}}=\frac{1}{2} x \cdot D u_{\alpha \bar{\beta}} .
$$

Differentiating $\Phi=\ln \operatorname{det}\left(u_{\alpha \bar{\beta}}\right)$,

$$
D \Phi=g^{\alpha \bar{\beta}} D u_{\alpha \bar{\beta}} .
$$

Combining these equations, we get

$$
g^{\alpha \bar{\beta}} \frac{\partial^{2} \Phi}{\partial z^{\alpha} \partial \bar{z}^{\beta}}=\frac{1}{2} x \cdot D \Phi .
$$

In particular, we have the important relation

$$
R=-\frac{1}{2} x \cdot D \Phi
$$

2.2. Inequality for scalar curvature $R$. Differentiating $R=-\frac{1}{2} x \cdot D \Phi$ twice and using $R_{\alpha \bar{\beta}}=-\frac{\partial^{2} \Phi}{\partial z^{\alpha} \partial \bar{z}^{\beta}}$,

$$
\frac{\partial^{2} R}{\partial z^{\alpha} \partial \bar{z}^{\beta}}=-\frac{\partial^{2} \Phi}{\partial z^{\alpha} \partial \bar{z}^{\beta}}-\frac{1}{2} x \cdot D \frac{\partial^{2} \Phi}{\partial z^{\alpha} \partial \bar{z}^{\beta}}=R_{\alpha \bar{\beta}}+\frac{1}{2} x \cdot D R_{\alpha \bar{\beta}} .
$$

Also, differentiating $R=g^{\alpha \bar{\beta}} R_{\alpha \bar{\beta}}$,

$$
D R=-g^{\alpha \bar{\gamma}} D u_{\bar{\gamma} \delta} g^{\delta \bar{\beta}} R_{\alpha \bar{\beta}}+g^{\alpha \bar{\beta}} D R_{\alpha \bar{\beta}} .
$$

Hence by (2.1),

$$
\begin{aligned}
\frac{1}{2} x \cdot D R & =-g^{\alpha \bar{\gamma}}\left(\frac{1}{2} x \cdot D u_{\bar{\gamma} \delta}\right) g^{\delta \bar{\beta}} R_{\alpha \bar{\beta}}+g^{\alpha \bar{\beta}} \frac{1}{2} x \cdot D R_{\alpha \bar{\beta}} \\
& =g^{\alpha \bar{\gamma}}\left(R_{\bar{\gamma} \delta}\right) g^{\delta \bar{\beta}} R_{\alpha \bar{\beta}}+g^{\alpha \bar{\beta}} \frac{1}{2} x \cdot D R_{\alpha \bar{\beta}} .
\end{aligned}
$$


Coupled with (2.4), we get

$$
g^{\alpha \bar{\beta}} \frac{\partial^{2} R}{\partial z^{\alpha} \partial \bar{z}^{\beta}}-\frac{1}{2} x \cdot D R=R-g^{\alpha \bar{\gamma}} g^{\delta \bar{\beta}} R_{\alpha \bar{\beta}} R_{\bar{\gamma} \delta} \leq R-\frac{1}{m} R^{2},
$$

or equivalently

$$
g^{\alpha \bar{\beta}} \frac{\partial^{2} R}{\partial z^{\alpha} \partial \bar{z}^{\beta}} \leq \frac{1}{2} x \cdot D R+R-\frac{1}{m} R^{2} .
$$

2.3. Inequality for distance $\rho$. Fix a point $x \in \mathbb{C}^{m}$, and let $\rho=\rho(x)$. We assume that $x$ is not in the cut locus of 0 . Since $\left(\mathbb{C}^{m}, g\right)$ is complete, there is a (unique) unit speed minimizing geodesic $\chi:[0, \rho] \rightarrow \mathbb{C}^{m}$ from 0 to $x$. We introduce a vector field $X(\tau)$ along $\chi(\tau)$ defined by $X=\chi^{\alpha} \frac{\partial}{\partial z^{\alpha}}+\chi^{\bar{\beta}} \frac{\partial}{\partial \bar{z}^{\beta}}$, where we regard $\chi \in \mathbb{C}^{m}$ as a tangent vector. Note that $X(0)=0$ and $X(\rho)=x^{i} \frac{\partial}{\partial x^{i}}$.

We proceed to compute the directional derivative $x \cdot D \rho(x)$ using the metric $g$ :

$$
\begin{aligned}
x \cdot D \rho(x) & =\left\langle X(\rho), \nabla_{g} \rho\right\rangle_{g}=\langle X(\rho), \dot{\chi}(\rho)\rangle \\
& =\int_{0}^{\rho} \frac{d}{d \tau}\langle X(\tau), \dot{\chi}(\tau)\rangle d \tau=\int_{0}^{\rho}\left\langle\nabla_{\tau} X(\tau), \dot{\chi}(\tau)\right\rangle d \tau,
\end{aligned}
$$

where the tangent vector $\dot{\chi}(\tau)=\frac{d}{d \tau} \chi$ and for simplicity of notation we have dropped the subscript $g$ in the inner product $\langle,\rangle_{g}$. To calculate the above integrand, we first compute the covariant derivative of $X$ along $\chi$ :

$$
\begin{aligned}
\nabla_{\tau} X & =\dot{\chi}^{\alpha} \frac{\partial}{\partial z^{\alpha}}+\dot{\chi}^{\bar{\beta}} \frac{\partial}{\partial \bar{z}^{\beta}}+\chi^{\alpha} \nabla_{\dot{\chi}} \frac{\partial}{\partial z^{\alpha}}+\chi^{\bar{\beta}} \nabla_{\dot{\chi}} \frac{\partial}{\partial \bar{z}^{\beta}} \\
& =\dot{\chi}+\chi^{\alpha} \Gamma_{\gamma \alpha}^{\mu} \dot{\chi}^{\gamma} \frac{\partial}{\partial z^{\mu}}+\chi^{\bar{\beta}} \Gamma_{\bar{\delta} \bar{\beta}}^{\bar{\nu}} \dot{\chi}^{\bar{\delta}} \frac{\partial}{\partial \bar{z}^{\nu}} .
\end{aligned}
$$

Then using the identity $\Gamma_{\gamma \alpha}^{\mu} g_{\mu \bar{\beta}}=u_{\gamma \alpha \bar{\beta}}$ (for a Kähler potential) and (2.1), we have

$$
\left\langle\nabla_{\tau} X, \dot{\chi}\right\rangle=1+X \cdot D u_{\alpha \bar{\beta}} \dot{\chi}^{\alpha} \dot{\chi}^{\bar{\beta}}=1-2 R_{\alpha \bar{\beta}} \dot{\chi}^{\alpha} \dot{\chi}^{\bar{\beta}} \text {. }
$$

Therefore, we have the formula:

$$
x \cdot D \rho(x)=\rho(x)-\int_{0}^{\rho} 2 R_{\alpha \bar{\beta}} \dot{\chi}^{\alpha} \dot{\chi}^{\bar{\beta}} d \tau .
$$

We have the following estimate for the Laplacian of the distance function $\rho$.

Lemma 2.1. Suppose Ric $\leq K$ on $B_{g}\left(0, \rho_{0}\right)$ for $\rho_{0}>0$. If $\rho(x)>\rho_{0}$ and $x$ is not in the cut locus of 0 , then

$$
g^{\alpha \bar{\beta}} \frac{\partial^{2} \rho}{\partial z^{\alpha} \partial \bar{z}^{\beta}}(x) \leq\left[\frac{2 m-1}{2 \rho_{0}}+\frac{1}{3} K \rho_{0}\right]+\frac{1}{2} x \cdot D \rho(x)-\frac{1}{2} \rho(x) .
$$

Proof. The mean curvature $H$ of the geodesic sphere $\partial B_{g}(0, \rho)$ with respect to the normal $\nabla_{g} \rho$ equals $\frac{-1}{2 m-1} \Delta_{g} \rho$. As calculated in [C, p.52], $H$ satisfies the following differential inequality

$$
H_{\rho} \geq H^{2}+\frac{1}{2 m-1} \operatorname{Ric}\left(\nabla_{g} \rho, \nabla_{g} \rho\right) .
$$

Let $H=\frac{-1}{\rho}+b$. Since the Riemannian metric $g$ is asymptotically Euclidean as $\rho \rightarrow$ 0 , we know $b$ is bounded for small $\rho$ (in fact $O(\rho)$ ). We then have a corresponding inequality for $b$ :

$$
\frac{1}{\rho^{2}}+b_{\rho} \geq \frac{1}{\rho^{2}}-2 \frac{1}{\rho} b+b^{2}+\frac{1}{2 m-1} \operatorname{Ric}\left(\nabla_{g} \rho, \nabla_{g} \rho\right),
$$


and consequently

$$
\left(\rho^{2} b\right)_{\rho} \geq \rho^{2} b^{2}+\frac{\rho^{2}}{2 m-1} \operatorname{Ric}\left(\nabla_{g} \rho, \nabla_{g} \rho\right) \geq \frac{\rho^{2}}{2 m-1} \operatorname{Ric}\left(\nabla_{g} \rho, \nabla_{g} \rho\right) .
$$

Integrating along $\chi(\tau)$, we arrive at

$$
b\left(\chi\left(\rho_{0}\right)\right) \geq \frac{1}{\rho_{0}^{2}} \int_{0}^{\rho_{0}} \frac{\tau^{2}}{2 m-1} \operatorname{Ric}(\dot{\chi}, \dot{\chi}) d \tau .
$$

Then for $\rho \geq \rho_{0}$,

$$
\begin{aligned}
H(\chi(\rho)) & =H\left(\chi\left(\rho_{0}\right)\right)+\int_{\rho_{0}}^{\rho} H_{\rho} d \tau \\
& \geq H\left(\chi\left(\rho_{0}\right)\right)+\int_{\rho_{0}}^{\rho} \frac{1}{2 m-1} \operatorname{Ric}(\dot{\chi}, \dot{\chi}) d \tau \\
& \geq \frac{-1}{\rho_{0}}+\frac{1}{\rho_{0}^{2}} \int_{0}^{\rho_{0}} \frac{\tau^{2}}{2 m-1} \operatorname{Ric}(\dot{\chi}, \dot{\chi}) d \tau+\int_{\rho_{0}}^{\rho} \frac{1}{2 m-1} \operatorname{Ric}(\dot{\chi}, \dot{\chi}) d \tau
\end{aligned}
$$

Substituting back $\Delta_{g} \rho=-(2 m-1) H$ and recalling $x=\chi(\rho)$, we obtain

$$
\Delta_{g} \rho \leq \frac{2 m-1}{\rho_{0}}-\int_{0}^{\rho} \operatorname{Ric}(\dot{\chi}, \dot{\chi}) d \tau+\int_{0}^{\rho_{0}}\left(1-\frac{\tau^{2}}{\rho_{0}^{2}}\right) \operatorname{Ric}(\dot{\chi}, \dot{\chi}) d \tau,
$$

when $\rho \geq \rho_{0}$. In fact, this estimate was first derived in $[\mathrm{P}$, Sec.8] (by a second variation argument).

Note that the Riemannian Laplacian $\Delta_{g}=2 g^{\alpha \bar{\beta}} \frac{\partial^{2}}{\partial z^{\alpha} \partial \bar{z}^{\beta}}$ and $\operatorname{Ric}(\dot{\chi}, \dot{\chi})=2 R_{\alpha \bar{\beta}} \dot{\chi}^{\alpha} \dot{\chi}^{\bar{\beta}}$. Then (2.7) follows from combining (2.6) with (2.8).

To prove Theorem 1.1 we will also need an inequality for $\rho^{y}(x)=$ distance from $x$ to $y$ in $\left(\mathbb{C}^{m}, g\right)$. Following the previous argument and using $\left(X-y^{i} \frac{\partial}{\partial x^{i}}\right)$ instead of $X$ for the vector field along $\chi$, we have

Lemma 2.2. Suppose Ric $\leq K$ on $B_{g}\left(y, \rho_{0}\right)$. Fix $A>\rho_{0}$, and let $x \in B_{g}(y, A)$. If $\rho^{y}(x)>\rho_{0}$ and $x$ is not in the cut locus of $y$, then

$$
g^{\alpha \bar{\beta}} \frac{\partial^{2} \rho^{y}}{\partial z^{\alpha} \partial \bar{z}^{\beta}}(x) \leq\left[\frac{2 m-1}{2 \rho_{0}}+\frac{1}{3} K \rho_{0}\right]+\frac{1}{2} x \cdot D \rho^{y}(x)-\frac{1}{2} \rho^{y}(x)+C_{0} A|y|,
$$

where the constant $C_{0}$ only depends on the "Euclidean" norms of $D u_{\alpha \bar{\beta}}$ and $g^{-1}$ in $B_{g}(y, A)$.

Proof. Arguing as above, let $\chi$ be the unit speed minimizing geodesic from $y$ to $x$. Using $\left(X-y^{i} \frac{\partial}{\partial x^{i}}\right)$ for the vector field along $\chi$ (note that $\left.X(0)=y^{i} \frac{\partial}{\partial x^{i}}\right)$, we have

$$
(x-y) \cdot D \rho^{y}(x)=\rho^{y}(x)-\int_{0}^{\rho^{y}(x)} 2 R_{\alpha \bar{\beta}} \dot{\chi}^{\alpha} \dot{\chi}^{\bar{\beta}} d \tau-\int_{0}^{\rho^{y}(x)} y \cdot D u_{\alpha \bar{\beta}} \dot{\chi}^{\alpha} \dot{\chi}^{\bar{\beta}} d \tau .
$$

The conclusion of the lemma follows, as above, from combining this equation with (2.8). 


\section{Proof of Theorem 1.1}

First we prove the scalar curvature $R \geq 0$ on complete $\left(\mathbb{C}^{m}, g\right)$. Choose a cutoff function $\phi$ such that $\phi \equiv 1$ on $[0,1], \phi \equiv 0$ on $[2, \infty), \phi^{\prime} \leq 0,\left|\phi^{\prime}\right| \leq C_{1} \phi^{1 / 2}$, and $\left|\phi^{\prime \prime}-2\left(\phi^{\prime}\right)^{2} / \phi\right| \leq C_{2} \phi^{1 / 2}$. For any small $\rho_{0}>0, K=K\left(\rho_{0}\right)$ can be chosen so that Ric $\leq K$ on $B_{g}\left(0,2 \rho_{0}\right)$. Fixing $A>\rho_{0}$, we derive an effective negative lower bound (3.1) for $R$ on $B_{g}(0, A)$. Set $\tilde{R}=\phi(\rho / A) R$. If $R<0$ at some point in $B_{g}(0,2 A)$, then $\tilde{R}$ achieves a negative minimum at some point $p \in B_{g}(0,2 A)$, as $\overline{B_{g}(0,2 A)}$ is compact for each $A>0$ by the completeness of $\left(\mathbb{C}^{m}, g\right)$. We consider two cases.

Case 1: $p$ is not in the cut locus of 0 . Then $\rho$ is smooth near $p$, and we have

$$
\Delta_{g} \tilde{R}=\left(\frac{\phi^{\prime \prime}}{A^{2}}+\frac{\phi^{\prime}}{A} \Delta_{g} \rho\right) R+\phi \Delta_{g} R+2\langle\nabla \phi, \nabla R\rangle,
$$

where we have used $|\nabla \rho|=1$. In order to have a linear differential inequality for $\tilde{R}$ with smooth coefficients (even for the Lipschitz function $\rho$ ), we rewrite

$$
\begin{aligned}
& \langle\nabla \phi, \nabla R\rangle=\left\langle\nabla \phi, \frac{\nabla \tilde{R}}{\phi}-\frac{\nabla \phi}{\phi^{2}} \tilde{R}\right\rangle=\left\langle\nabla \frac{\tilde{R}}{R}, \frac{\nabla \tilde{R}}{\phi}\right\rangle-\frac{|\nabla \phi|^{2}}{\phi^{2}} \tilde{R} \\
& \stackrel{|\nabla \rho|=1}{=} \frac{|\nabla \tilde{R}|^{2}}{\tilde{R}}-\left\langle\frac{\nabla R}{R}, \nabla \tilde{R}\right\rangle-\frac{\left(\phi^{\prime}\right)^{2}}{A^{2} \phi} R \\
& \stackrel{\tilde{R}<0}{\leq}-\left\langle\frac{\nabla R}{R}, \nabla \tilde{R}\right\rangle-\frac{\left(\phi^{\prime}\right)^{2}}{A^{2} \phi} R .
\end{aligned}
$$

Using $\Delta_{g}=2 g^{\alpha \bar{\beta}} \frac{\partial^{2}}{\partial z^{\alpha} \partial \bar{z}^{\beta}}$, the inequalities (2.7) and (2.5) for $\rho$ and $R$, and the inequalities for $\phi$, we get

$$
\begin{aligned}
& g^{\alpha \bar{\beta}} \frac{\partial^{2} \tilde{R}}{\partial z^{\alpha} \partial \bar{z}^{\beta}} \stackrel{\phi^{\prime} R>0}{\leq} \quad \frac{1}{2 A^{2}}\left[\phi^{\prime \prime}-\frac{2\left(\phi^{\prime}\right)^{2}}{\phi}\right] R+\frac{\phi^{\prime} R}{A}\left[\left(\frac{2 m-1}{2 \rho_{0}}+\frac{1}{3} K \rho_{0}\right)+\frac{1}{2} x \cdot D \rho(x)\right] \\
&+\phi\left(\frac{1}{2} x \cdot D R+R-\frac{1}{m} R^{2}\right)-\left\langle\frac{\nabla R}{R}, \nabla \tilde{R}\right\rangle \\
& \leq \quad \frac{C_{2}}{2 A^{2}} \phi^{1 / 2}|R|+\frac{C_{1}}{A}\left(\frac{2 m-1}{2 \rho_{0}}+\frac{1}{3} K \rho_{0}\right) \phi^{1 / 2}|R|-\frac{\phi R^{2}}{m} \\
&+\tilde{R}+\frac{1}{2} x \cdot D \tilde{R}-\left\langle\frac{\nabla R}{R}, \nabla \tilde{R}\right\rangle \\
& \leq \frac{C\left(m, \rho_{0}\right)}{A^{2}}+\tilde{R}+b(x) \cdot D \tilde{R},
\end{aligned}
$$

where $b(x)$ is a smooth function and $C\left(m, \rho_{0}\right)$ is a constant that depends only on $m, \rho_{0}, C_{1}$, and $C_{2}$. Since $\tilde{R}$ achieves its minimum at $p$, we have $\tilde{R}(p) \geq-\frac{C\left(m, \rho_{0}\right)}{A^{2}}$ and $R \geq-\frac{C\left(m, \rho_{0}\right)}{A^{2}}$ on $B_{g}(0, A)$.

Case 2: $p$ is in the cut locus of 0 . Then $\rho$ is not smooth at $p$, and we argue using Calabi's trick [C, p.53] of approximating $\rho$ from above by smooth functions (cf. [CCG1, pp.453-456]). For completeness, we include the argument here. Let $\chi$ be a unit speed geodesic from 0 to $p$ that minimizes length, and define $\rho_{\varepsilon}=\rho^{\chi(\varepsilon)}+\varepsilon$, where $\rho^{\chi(\varepsilon)}$ is the distance to $\chi(\varepsilon)$. Then $\rho_{\varepsilon}(p)=\rho(p)$ and $\rho_{\varepsilon} \geq \rho$ near $p$. Since $p$ is not in the cut locus of $\chi(\varepsilon)$, we know that $\rho_{\varepsilon}$ is smooth near $p$. Let $\tilde{R}_{\varepsilon}=\phi\left(\rho_{\varepsilon} / A\right) R$. 
Then $\tilde{R}_{\varepsilon}$ is smooth near $p$. Furthermore, since $\phi$ is decreasing and $R<0$ near $p$, the above properties of $\rho_{\varepsilon}$ show that $\tilde{R}_{\varepsilon}(p)=\tilde{R}(p)$ and $\tilde{R}_{\varepsilon} \geq \tilde{R}$ near $p$. It follows that $\tilde{R}_{\varepsilon}$ has a local minimum at $p$. Arguing as we did in Case 1, and using Lemma 2.2 to estimate $\rho_{\varepsilon}$, we have

$$
g^{\alpha \bar{\beta}} \frac{\partial^{2} \tilde{R}_{\varepsilon}}{\partial z^{\alpha} \partial \bar{z}^{\beta}} \leq \frac{C\left(m, \rho_{0}\right)}{A^{2}}+\tilde{R}_{\varepsilon}+b(x) \cdot D \tilde{R}_{\varepsilon}+\frac{\phi^{\prime} R}{A}\left[C_{0} 2 A|\chi(\varepsilon)|\right],
$$

where $b(x)$ is a smooth function, $C\left(m, \rho_{0}\right)$ is a constant that depends only on $m$, $\rho_{0}, C_{1}$, and $C_{2}$, and $C_{0}$ is a constant depending on the "Euclidean" norms of $D u_{\alpha \bar{\beta}}$ and $g^{-1}$ in $\overline{B_{g}(0,2 A)}$. Note that we may choose $C_{0}$ independent of (small) $\varepsilon$. At $p$ we have

$$
\tilde{R}_{\varepsilon}(p) \geq-\frac{C\left(m, \rho_{0}\right)}{A^{2}}-\frac{\phi^{\prime} R}{A}(p)\left[C_{0} 2 A|\chi(\varepsilon)|\right] .
$$

Taking $\varepsilon \rightarrow 0$, we arrive at the inequality $\tilde{R}(p) \geq-\frac{C\left(m, \rho_{0}\right)}{A^{2}}$, which shows $R \geq$ $-\frac{C\left(m, \rho_{0}\right)}{A^{2}}$ on $B_{g}(0, A)$.

Combining Case 1 and Case 2, we have shown that

$$
R \geq-\frac{C\left(m, \rho_{0}\right)}{A^{2}} \text { on } B_{g}(0, A) .
$$

Taking $A \rightarrow \infty$, we arrive at $R \geq 0$ on $\mathbb{C}^{m}$.

Now, we finish the proof of Theorem 1.1. Since $R \geq 0$, it follows from the equation $R=-g^{\alpha \bar{\beta}} \frac{\partial^{2} \Phi}{\partial z^{\alpha} \partial \bar{z}^{\beta}}=-\frac{1}{2} x \cdot D \Phi$ that $\Phi$ achieves its global maximum at the origin. Applying the strong maximum principle to equation (2.2) we conclude that $\Phi$ is constant. Using $\frac{1}{2} x \cdot D u-u=\Phi$, we have

$$
\frac{1}{2} x \cdot D[u+\Phi(0)]=u+\Phi(0) .
$$

Finally, it follows from Euler's homogeneous function theorem that smooth $u+$ $\Phi(0)$ is a homogeneous order two polynomial.

Remark. In fact, one sees that the Lipschitz function $\tilde{R}$ (on the set where $\tilde{R}<0)$ is a subsolution to

$$
g^{\alpha \bar{\beta}} \frac{\partial^{2} \tilde{R}}{\partial z^{\alpha} \partial \bar{z}^{\beta}} \leq \frac{C\left(m, \rho_{0}\right)}{A^{2}}+\tilde{R}+b(x) \cdot D \tilde{R}
$$

in the viscosity sense by using the same trick of Calabi. It follows from the comparison principle (cf. [CIL, p.18]) that the same negative lower bounds for $\tilde{R}$ and hence $R$ can be derived.

\section{REFERENCES}

[C] Calabi, E., An extension of E. Hopf's maximum principle with an application to Riemannian geometry, Duke Math. J. 25 (1958), 45-56.

[CCY] Chau, A., Chen, J., Yuan, Y., Rigidity of entire self-shrinking solutions to curvature flows, J. Reine Angew. Math. 664 (2012), 229-239.

[Ch] Chen, B.-L., Strong uniqueness of the Ricci flow, J. Differential Geom. 82 (2009), no. 2, 363-382.

[CCG1] Chow, B., Chu, S.-C., Glickenstein, D., Guenther, C., Isenberg, J., Ivey, T., Knopf, D., Lu, P., Luo, F., Ni, L., Ricci flow: techniques and applications, part I: geometric aspects, Math. Surv. Monogr. 135. Amer. Math. Soc., 2007.

[CCG4] Chow, B., Chu, S.-C., Glickenstein, D., Guenther, C., Isenberg, J., Ivey, T., Knopf, D., Lu, P., Luo, F., Ni, L., Ricci flow: techniques and applications, part IV: long time solutions and related topics. To appear. 
[CIL] Crandall, M.G., Ishii, H., Lions, P., User's guide to viscosity solutions of second order partial differential equations, Bull. Amer. Math. Soc. 27 (1992), no. 1, 1-67.

[DX] Ding, Q., Xin, Y.L., The rigidity theorems for Lagrangian self shrinkers, J. Reine Angew. Math. DOI: 10.1515/crelle-2012-0081.

[HW] Huang, H., Wang, Z., On the entire self-shrinking solutions to Lagrangian mean curvature flow, Calc. Var. Partial Differential Equations 41 (2011), no. 3-4, 321-339.

[P] Perelman, G., The entropy formula for the Ricci flow and its geometric applications. arXiv:math/0211159

Department of Mathematics, Box 354350, University of Washington, Seattle, WA 98195

E-mail address: drugan@math.washington.edu

Department of Mathematics, University of Oregon, Eugene, OR 97403

E-mail address: penglu@uoregon.edu

Department of Mathematics, Box 354350, University of Washington, Seattle, WA 98195

E-mail address: yuan@math.washington.edu 